\title{
Spectral cues provided by the pinna for monaural localization in the horizontal plane
}

\author{
ROSEMARY FLANNERY and ROBERT A. BUTLER \\ Departments of Surgery (Otolaryngology) and Behavioral Sciences \\ The University of Chicago, Chicago, Illinois 60637
}

\begin{abstract}
Six subjects located, monaurally, 1.0-kHz-wide noise bursts whose source originated on the side of the functioning ear and whose center frequency ranged from 4.0 through $9.0 \mathrm{kHz}$ (Part 1). Irrespective of their actual locations, the stimuli appeared to migrate from the frontal sector of the arc toward the side as the center frequency was increased above $4.0 \mathrm{kHz}$. For some subjects, the sounds appeared again in front at the higher center frequencies. Comparable data were obtained with noise bursts $2.0 \mathrm{kHz}$ in width. We referred to these constellations of location judgments, influenced by the frequency composition of the stimuli, as spatial referent maps. In Part 2, we measured, by means of a miniature microphone placed at the entrance of the external ear canal, the pinna amplification function for these same stimuli emanating from the same locations. The results showed a positive relation between the apparent location of noise bursts centered at $6.0 \mathrm{kHz}$ and above and the relative amplification provided by the pinna. Localization performances by two subjects, chosen on the basis of their noncorresponding spatial referent maps, were examined for stimuli of wider bandwidths (Part 3). Their proficiency differed markedly from one another, which we accounted for in terms of different spatial referent maps that were associated with differences in the pinna amplification function.
\end{abstract}

When binaural differences in stimulus intensity and arrival time are unavailable, listeners depend largely on spectral cues for locating sounds. A moment's reflection will assure one that these situations are ubiquitous. In numerous minute-to-minute transactions with the environment, only the ear nearest the sound source is stimulated at suprathreshold levels. Hence, to make a location judgment that is more precise than mere sidedness, we must rely on spectral cues. In fact, we continually utilize them to judge the elevation of sounds. Spectral cues are furnished primarily by the pinna (see Butler, 1975, for a review of this literature), and we know that in the sagittal plane, a trough in the sound's spectrum migrates to lower frequency regions as the height of the sound sources is lowered (Bloom, 1977; Butler \& Belendiuk, 1977; Hebrank \& Wright, 1974).

A recent paper by Butler and Flannery (1980) represents a beginning in the quest to piece together the spectral components implicated in monaural localization in the horizontal plane. First, we found that the apparent location of a $1.0-\mathrm{kHz}$-wide noise burst moved from near the front toward the side of the functioning ear as the center frequency (CF) was increased from 4.0 to about $8.0 \mathrm{kHz}$. Then, when the CF was raised from approximately 8.0 to around $12.0 \mathrm{kHz}$, the migration of the sound's apparent location again moved from front to side. With a CF of

This work was supported by U.S. Public Health Service Grant NS-11154-04.
13.0 and $14.0 \mathrm{kHz}$, the sound, for some listeners, appeared to originate once more from the front. We referred to the orderly changes of apparent location associated with changes in CF as spatial referent maps (SRMs) and numbered them SRM 1 (4.0$8.0 \mathrm{kHz})$, SRM $2(8.0-12.0 \mathrm{kHz})$, and the start of SRM $3(13.0 \mathrm{kHz})$, respectively. These frequency ranges are approximate. Next, we sought to find out how these SRMs contributed to proficient monaural localization. Time and again, we noted that a noise burst of fixed bandwidth (BW) could be located more accurately if its frequency composition connected SRMs rather than being confined to a single SRM. The difference was sometimes so dramatic that we assumed the "map connecting" aspect of the stimulus to be fundamental to the precision with which it can be located.

Our plan for the present study was to measure the relative amplification provided by the pinna for narrow noise bands differing both in location and $C F$ and to look for a correspondence between the pattern of amplification and the apparent location of these stimuli. We first established (Part 1) SRMs for each listener, employing a much larger number of stimulus presentations than had been used previously. In Part 2, we collected data on each person's pinna amplification function for those same stimuli. For the third part of our study, we selected two subjects and attempted to interrelate their SRMs, their pinna amplification function, and their ability to locate a wider noise band. 


\section{PART 1: THE ESTABLISHMENT OF SRMs}

\section{Method}

Six subjects participated, four of whom served in the earlier study (Butler \& Flannery, 1980). To create a monaural listening condition, a Mine Safety Appliance (MSA) ear defender was inserted in the right external ear canal and a MSA circumaural muff was placed snugly over the right pinna and strapped to the head. Noise bursts, whose BW was $1.0 \mathrm{kHz}$, were presented from loudspeakers (KLH: 4 in. diam.) positioned $15 \mathrm{deg}$ apart at azimuthal locations ranging from 270 through $345 \mathrm{deg}$-positions that extended along the arc on the side of the open (unoccluded) ear. No stimuli originated from straight-ahead, since normal-hearing persons with one ear occluded nearly always fail to locate a sound emanating from that position. They displace it toward the open ear. The noise bursts were centered at 4.0, 5.0,6.0, 7.0, 8.0, and $9.0 \mathrm{kHz}$. CFs higher than $9.0 \mathrm{kHz}$ were not used because the output of the microphone that we employed to assess the amplification afforded by the pinna (Knowles Electronics, Model BL 1685) dropped precipitously at $9.5 \mathrm{kHz}$. We constructed the noise bands by setting the tonal stimulus at the desired $\mathrm{CF}$ and modulating it by a $.5-\mathrm{kHz}$ low-pass noise whose high-frequency cutoff was $36 \mathrm{~dB} /$ octave. Rise-fall time was $10 \mathrm{msec}$; duration was $30 \mathrm{msec}$; and repetition rate was approximately $3 / \mathrm{sec}$. Loudness level averaged $20 \mathrm{~dB}$ SL re noise bursts originating from the loudspeaker near the middle of the array (viz., the loudspeaker stationed at $\mathbf{3 1 5}$ deg azimuth). Following a prearranged plan, we varied the intensity $\pm 2 \mathrm{~dB}$ aperiodically.

Testing took place in a room, $15 \times 13 \frac{1}{2} \times 8 \frac{1}{2} \mathrm{ft}$., soundtreated by means of sound-absorbent material on the walls and ceiling and carpeting on the floor. The electronic equipment for generating and controlling the stimuli was housed in an adjacent room. Subjects were seated in the center of the room, $5 \mathrm{ft}$. removed from the loudspeaker array. The array was numbered consecutively from 1 through 6 , with 1 being stationed at 345 deg azimuth and 6 at 270 deg. Fixed to the back of the subject's chair was an adjustable headrest; subjects were instructed to press their heads firmly against it throughout the test session. They were required to call out, via an intercom, the number of the loudspeaker from which the sounds appeared. The following constraints were placed on the formation of stimulus presentation order: Within a block of 60 consecutive trials, each loudspeaker was activated 10 times; each differently centered noise burst was presented 10 times; and no loudspeaker or CF was presented more than twice in succession. Given these constraints, the differently centered noise bursts and loudspeaker positions were then chosen randomly. In all, 360 trials were given to each listener. Four test sessions, lasting approximately $45 \mathrm{~min}$ apiece, were required to complete the establishment of the SRMs. To gain more information on the size of the BW suitable for generating orderly SRMs, we repeated the procedure with BWs of $2.0 \mathrm{kHz}$ and centered at $4.5,5.5,6.5,7.5$, and $8.5 \mathrm{kHz}$. The constraints on stimulus presentation order, mentioned above, applied to this series of trials as well, but now the differently centered noise bursts were presented 12 times within a block of 60 consecutive trials. Again, testing was continued over four test sessions.

\section{Results}

Table 1 shows the percent of trials that each azimuthal position was chosen as the origin of noise bursts $1.0 \mathrm{kHz}$ wide and centered at $4.0,5.0,6.0$, $7.0,8.0$, and $9.0 \mathrm{kHz}$. Table 2 lists the data on location judgments for noise bursts $2.0 \mathrm{kHz}$ in width and centered at $4.5,5.5,6.5,7.5$, and $8.5 \mathrm{kHz}$. A chisquare test carried out on each listener's data indicated a distribution of location judgments that differed significantly from chance beyond the .001 level of confidence for both the $1.0-\mathrm{kHz}$ - and $2.0-\mathrm{kHz}$-wide noise bands. The basis for these significant deviations from chance was that the apparent location of the noise bursts was influenced systematically by

Table 1

Percent of Loudspeaker Choices as a Function of the Center Frequency of a 1.0-kHz-Wide Noise Band

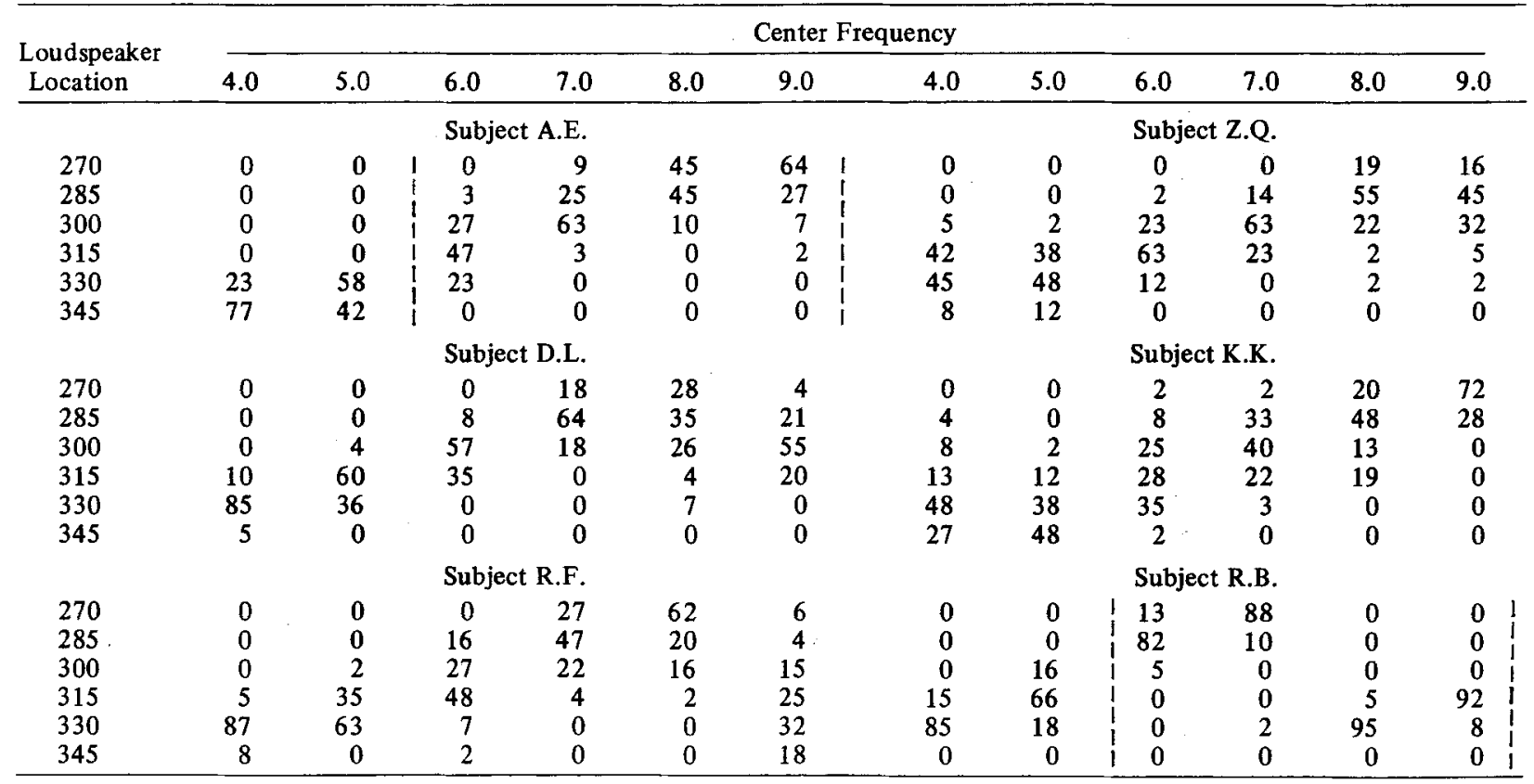

Note-The dashed vertical lines are explained in Part 3 (Method). 
Table 2

Percent of Loudspeaker Choices as a Function of the Center Frequency of a $2.0-\mathbf{k H z}$-Wide Noise Band

\begin{tabular}{|c|c|c|c|c|c|c|c|c|c|c|}
\hline \multirow{2}{*}{$\begin{array}{c}\text { Loudspeaker } \\
\text { Location }\end{array}$} & \multicolumn{10}{|c|}{ Center Frequency } \\
\hline & 4.5 & 5.5 & 6.5 & 7.5 & 8.5 & 4.5 & 5.5 & 6.5 & 7.5 & 8.5 \\
\hline & \multicolumn{5}{|c|}{ Subject A.E. } & \multicolumn{5}{|c|}{ Subject Z.Q. } \\
\hline 270 & 0 & 0 & 6 & 22 & 75 & 0 & 0 & 0 & 19 & 42 \\
\hline 285 & 0 & 0 & 8 & 51 & 21 & 0 & 0 & 6 & 63 & 44 \\
\hline 300 & 0 & 0 & 71 & 26 & 4 & 0 & 11 & 75 & 18 & 13 \\
\hline 315 & 6 & 32 & 14 & 1 & 0 & 60 & 65 & 18 & 0 & 0 \\
\hline 330 & 57 & 67 & 1 & 0 & 0 & 39 & 24 & 1 & 0 & 0 \\
\hline \multirow[t]{2}{*}{345} & 37 & 1 & 0 & 0 & 0 & 1 & 0 & 0 & 0 & 1 \\
\hline & \multicolumn{5}{|c|}{ Subject D.L. } & \multicolumn{5}{|c|}{ Subject K.K. } \\
\hline 270 & 0 & 0 & 32 & 18 & 5 & 0 & 0 & 0 & 12 & 57 \\
\hline 285 & 0 & 3 & 54 & 48 & 19 & 0 & 0 & 8 & 35 & 37 \\
\hline 300 & 0 & 26 & 14 & 31 & 63 & 10 & 8 & 22 & 29 & 3 \\
\hline 315 & 43 & 68 & 0 & 3 & 13 & 15 & 24 & 42 & 24 & 3 \\
\hline 330 & 57 & 3 & 0 & 0 & 0 & 32 & 44 & 27 & 0 & 0 \\
\hline \multirow[t]{2}{*}{345} & 0 & 0 & 0 & 0 & 0 & 43 & 24 & 1 & 0 & 0 \\
\hline & \multicolumn{5}{|c|}{ Subject R.F. } & \multicolumn{5}{|c|}{ Subject R.B. } \\
\hline 270 & 0 & 0 & 25 & 65 & 12 & 0 & 3 & 88 & 31 & 0 \\
\hline 285 & 0 & 0 & 51 & 29 & 14 & 0 & 76 & 12 & 3 & 0 \\
\hline 300 & 0 & 6 & 24 & 6 & 19 & 4 & 21 & 0 & 0 & 0 \\
\hline 315 & 1 & 71 & 0 & 0 & 24 & 71 & 0 & 0 & 0 & 3 \\
\hline 330 & 99 & 23 & 0 & 0 & 3 & 25 & 0 & 0 & 35 & 96 \\
\hline 345 & 0 & 0 & 0 & 0 & 28 & 0 & 0 & 0 & 31 & 1 \\
\hline
\end{tabular}

their frequency composition. Apparent location changed from the frontal sector of the arc (345 and $330 \mathrm{deg})$ toward the side $(270 \mathrm{deg})$ as the CF was increased beyond $4.0 \mathrm{kHz}$. Then, for some subjects (R.B., R.F., D.L., and Z.Q.), the stimuli appeared to shift, with varying degrees of abruptness, toward the frontal sector at the higher CFs. Only one listener (R.B.) located noise bursts at an accuracy exceeding that expected by chance, and this was for the $2.0-\mathrm{kHz}$ wide stimulus (75 correct responses in 360 trials; $\mathrm{p}<.025$, one-tailed distribution). Upon closer inspection of the data, we detected the main contribution to this better-than-chance performance, to wit: When the $2.0-\mathrm{kHz}$-wide noise band was centered at $7.5 \mathrm{kHz}, 28$ of 72 stimulus presentations were located correctly ( $p<.005$, one-tailed distribution). Of interest is that the correct responses were associated with the frontal and side azimuthal locations of the loudspeakers. Subject R.B.'s number of correct responses for the four other differently centered noise bands did not exceed chance expectation.

\section{PART 2: RELATIVE AMPLIFICATION PROVIDED BY THE PINNA}

\footnotetext{
Method

We compared the stimulus intensity necessary to attain a fixed output from the miniature microphone when positioned in the external ear canal and when positioned in space. Subjects were seated in the same place as they were during those sessions devoted to forming SRMs. We fitted the microphone to a MSA ear defender and inserted the defender into the left external ear canal. The diaphragm of the microphone faced outward. We took care to place the assembly such that the microphone was at the entrance to the ear
}

canal and cautioned subjects to keep their heads firmly against a headrest while the sound was on. The stimuli were the same as those used to delineate the SRMs $-1.0 \mathrm{kHz}$ wide and centered at 4.0 through $9.0 \mathrm{kHz}$ in steps of $1.0 \mathrm{kHz}$. They emanated from a loudspeaker placed successively at $345,330,315,300,285$, and $270 \mathrm{deg}$ azimuth. The output of the ear canal microphone was amplified (Hewlett-Packard Amplifier, Model 465A) and delivered to a VTVM. We manipulated the output of the loudspeaker in steps of $1 \mathrm{~dB}$ until a predetermined reading (reference value) was attained on the VTVM. The value was only $6 \mathrm{~dB}$ above the noise level, since we wished to avoid higher intensity levels that could distort the signal.

The first set of measurements was carried out with the microphone in the ear canal. Then we fixed the microphone to a lever, which we attached to the headrest, and maneuvered the lever so that the microphone was at nearly the same position and angle as it was when inserted in the subject's ear canal. Decibel reading necessary to reach VTVM reference value for each stimulus at each azimuthal position was recorded. This procedure was followed on three additional sets of measurements taken on subsequent days, and the attenuator readings required to reach our VTVM reference value were averaged for the microphone in the ear canal and for the microphone in space. The difference in intensity between the two mean values was taken as the relative amplification provided by the pinna for the differently centered $1.0-\mathrm{kHz}$-wide noise bands originating at each of the loudspeaker locations. The same operations were followed for arriving at an estimate of the pinna amplification function for the $2.0-\mathrm{kHz}$-wide noise bursts.

\section{Results}

Figures 1, 2, and 3 show individual data, for the 1.0-kHz-wide noise bands, on the association between the relative amplification furnished primarily by the pinna and the apparent location of the stimuli. $\mathrm{CF}$ serves as the parameter. The number above each point on the curves represents the percent of trials on which that loudspeaker location was chosen as the 


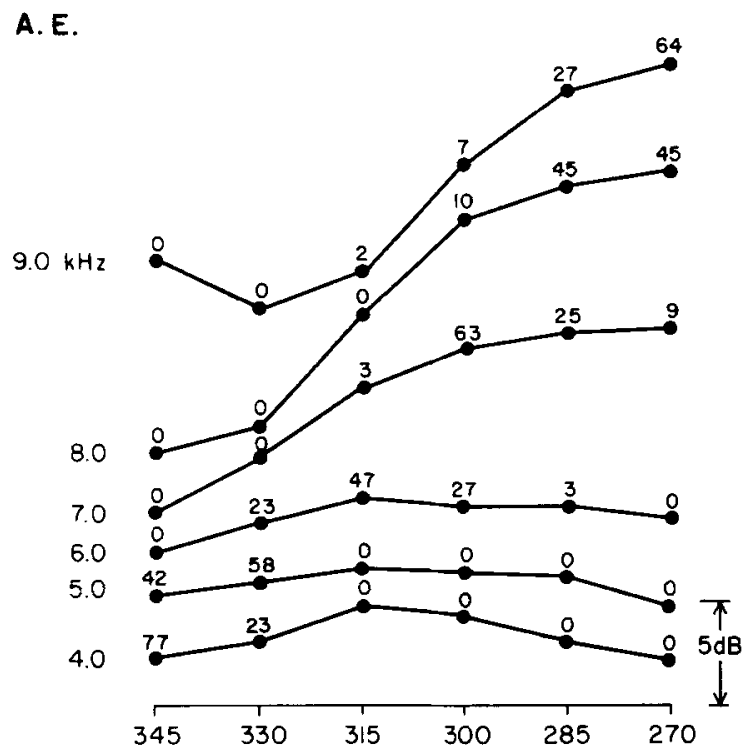

Z. Q.

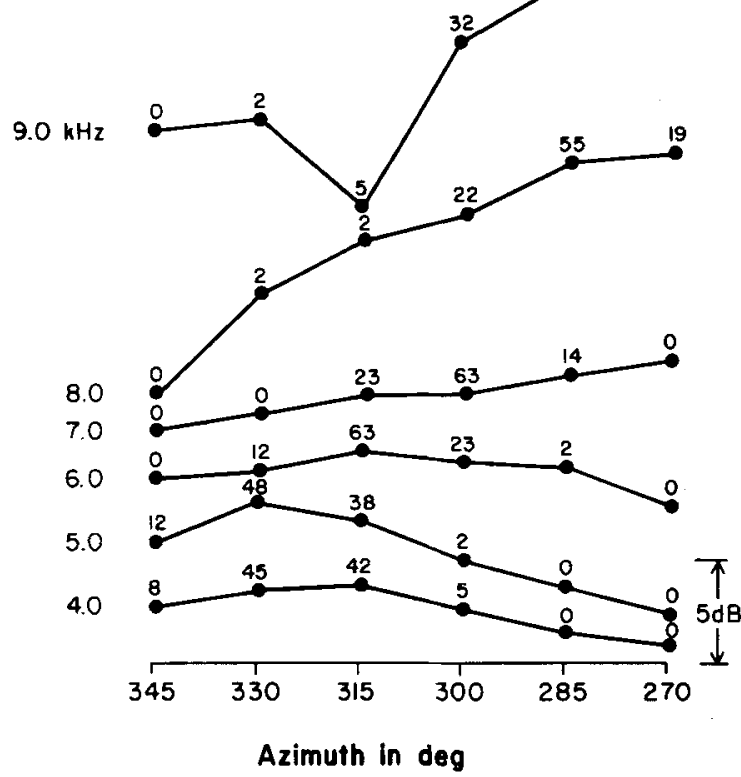

Figure 1. The pinna's relative amplification as a function of the azimuthal position of the sound source. CF of the $1.0-\mathrm{kHz} \mathrm{BW}$ serves as the parameter. Numbers above each point on the curves represent the percent of the trials on which that particular loudspeaker was chosen as the sound's source. Initials at upper left of each family of curves identify the subject.

place of the sound's origin. No direct relation between the two events is observable; that is, the apparent location of the sound does not necessarily correspond to the place at which the relative amplification is greatest. Yet, as CF of the noise bursts is increased, one can detect a shift in the pattern of the pinna amplification function that parallels a shift in the apparent location of the sound. Generally speaking, as the sounds, progressively higher in $C F$, ap- peared to move toward $270 \mathrm{deg}$, the amplification of stimuli actually emanating from loudspeakers stationed at 285 and $270 \mathrm{deg}$ was increasingly enhanced relative to that of those same stimuli when generated by loudspeakers positioned at 345 and $330 \mathrm{deg}$ azimuth.

Figure 4 illustrates this general correspondence for BWs of 1.0 and $2.0 \mathrm{kHz}$. To construct this figure, we first calculated the mean location judgments in degrees azimuth for each subject when responding to each differently centered BW, a procedure that generated 36 individual means -6 subjects $\times 6$ different stimuli. We next sorted out these mean location judgments into one of four contiguous regions along the horizontal plane: Region 1, 315 deg or greater; $\mathrm{Re}$ gion 2, 300 through 314 deg; Region 3, 285 through

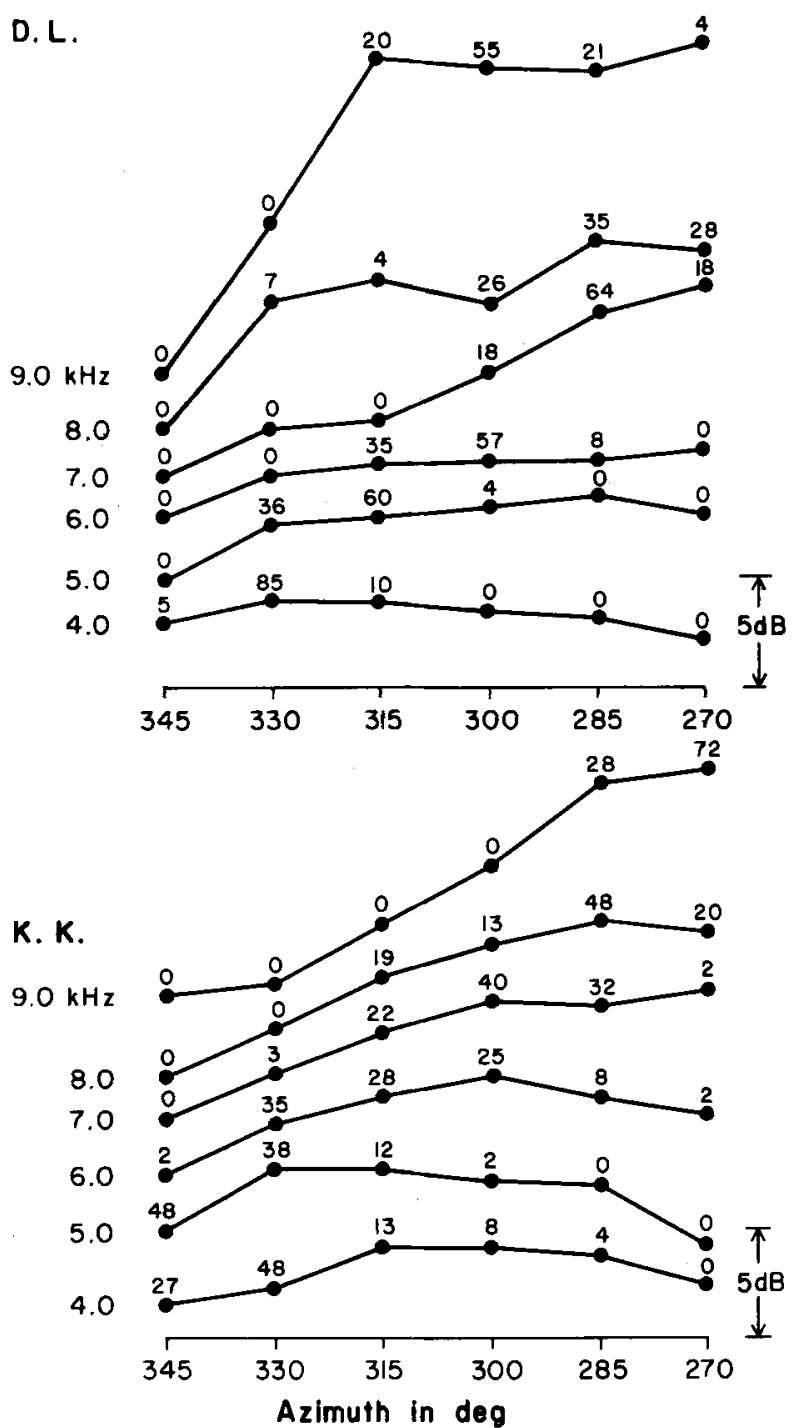

Figure 2. Relative amplificalion function of the pinna for Subjects D.L. and K.K. See Figure 1 for more details. 


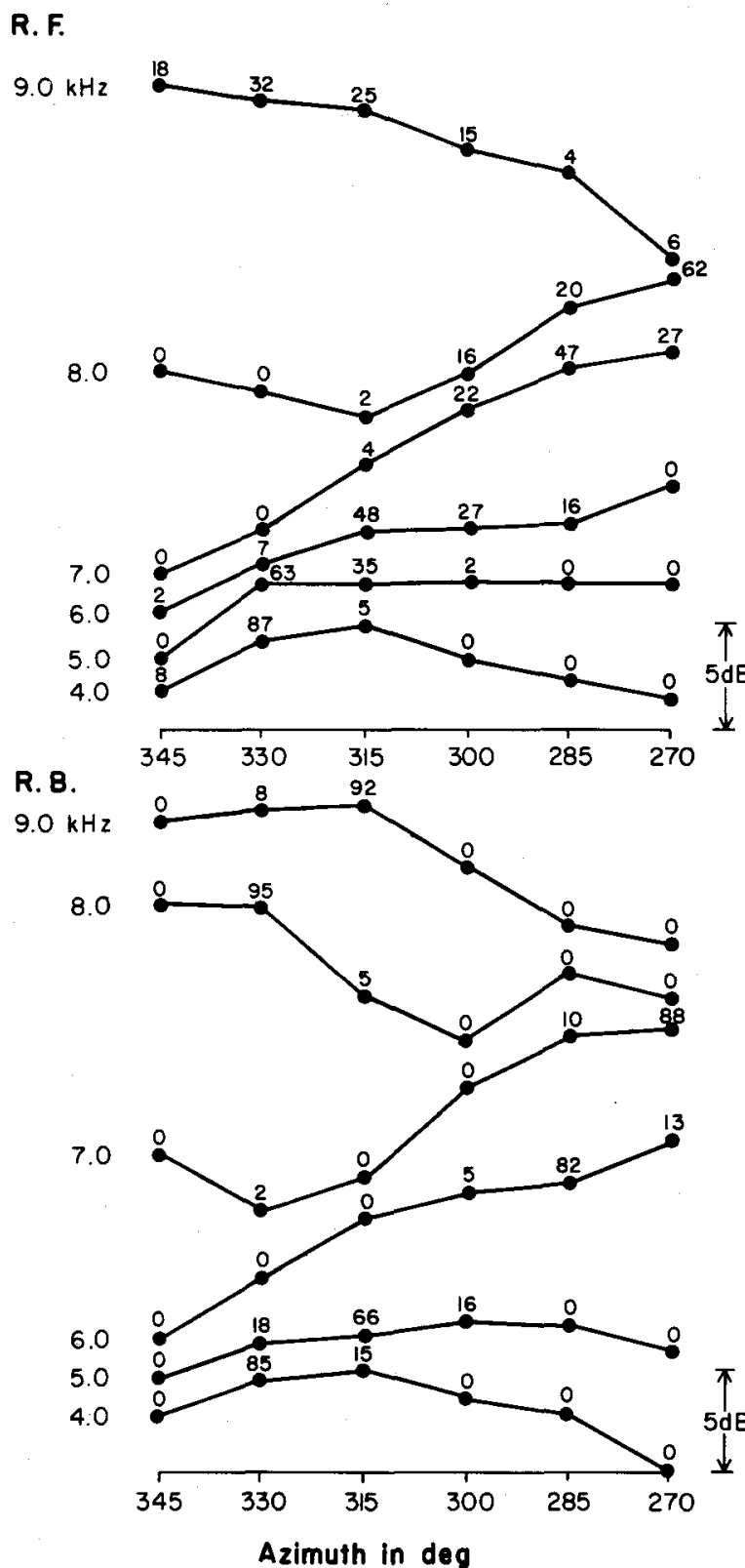

Figure 3. Relative amplification function of the pinna for Subjects R.F. and R.B. See Figure 1 for more details.

299 deg; and Region 4, less than 285 deg. Then, for each region, we averaged over subjects the pinna amplification curves associated with the respective location judgments. Location judgments fell within Region 1 when the $C F$ was 4.0 and $5.0 \mathrm{kHz}$ and, again, for Subjects R.F. and R.B., when the CF was 8.0 and/or $9.0 \mathrm{kHz}$. For these two situations, we made separate calculations. The closed circles-solid lines of Figure 4 represent the mean pinna amplification curves for the $1.0-\mathrm{kHz}$ BWs. Of the 36 mean location judgments, $11,7,9$, and 6 fell within Regions 1 through 4 , respectively. The open circles-dashed lines repre- sent the location judgments encompassed by Region 1 when the spectral composition of the noise bursts was centered at 8.0 and/or $9.0 \mathrm{kHz}$-the remaining three mean location judgments. Note that for these three judgments, the pinna amplification curve is inverted; that is, the amplification of sounds actually emanating from 345 and 330 deg now becomes greater relative to those that came from loudspeakers placed at 285 and 270 deg azimuth. This is seen more clearly in the individual data of R.F. and R.B. displayed in the previous figure (Figure 3); the transition in the form of the curves takes place between CFs of 8.0 and $9.0 \mathrm{kHz}$ for R.F. and between CFs of 7.0 and $8.0 \mathrm{kHz}$ for R.B.

Defining Regions 1 through 4 as we did above and distributing mean location judgments accordingly, we next sought to find out how closely the mean pinna amplification curves for noise bursts $2.0 \mathrm{kHz}$ in width would parallel those curves for the $1.0-\mathrm{kHz}-$ wide noise bursts. Here, the data consist of 30 mean location judgments and their associated pinna amplification curves -6 subjects $\times 5$ differently centered noise bursts. The results are represented by the closed triangles-solid lines. Numbers of mean location judgments falling within Regions 1 through 4 were 10,5 , 7 , and 6 , respectively. Unquestionably, the curves for the different BWs correspond closely to one another. The open triangles-dashed lines represent the pinna amplification function for higher stimulus CFs when
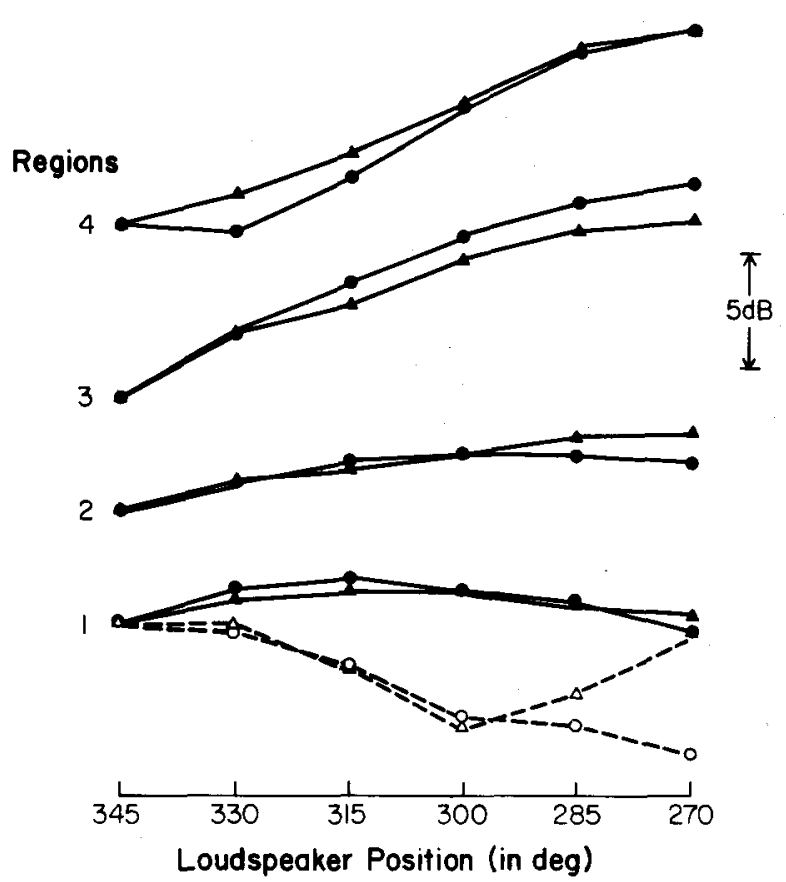

Figure 4. Group mean data on relative amplification of the pinna as a function of aximuthal position of the sound source. Regions 1 through 4 refer to location judgments confined to four different segments along the horizontal plane, as described in text. 
the mean location judgments fell again into Region 1 , as they did in two cases.

\section{PART 3: SPECIAL MONAURAL LOCALIZATION TESTS}

\section{Method}

These tests were carried out to further investigate the relation between localization accuracy and the frequency composition of the stimulus. Serving as the stimulus was a noise band centered at $7.5 \mathrm{kHz}$ and $4.0 \mathrm{kHz}$ wide. Such a stimulus can be arbitrarily partitioned into four contiguous bands of noise, each $1.0 \mathrm{kHz}$ wide and centered at $6.0,7.0,8.0$, and $9.0 \mathrm{kHz}$, respectively. And, as reported by Butler and Flannery (1980) and confirmed in Part 1 of this study, the localization judgments of each $1.0-\mathrm{kHz}$-wide noise band will differ depending on its CF. What we did in this study was to choose two listeners, A.E. and R.B., whose location judgments for the $1.0-\mathrm{kHz}$-wide noise bands differed from one another in a special way. Namely, A.E.'s location judgments for these stimuli changed from front to side as the CF was increased from 4.0 to $9.0 \mathrm{kHz}$ (see the distribution of her judgments encompassed by the dashed-vertical lines in Table 1). R.B.'s location judgments changed from front to side and returned abruptly to front as the CF was progressively increased (see his distribution of judgments confined by the dashed-vertical lines in Table 1). We were led to expect by the earlier report that R.B.'s localization performance would surpass that of A.E., even though the frequency composition of the stimulus was the same for both listeners, the reason being that a sound whose frequency composition overlaps adjacent SRMs can be more accurately localized than one whose frequency composition is restricted to a single SRM. As Table 1 shows, the former condition applies to R.B.'s data, the latter to A.E.'s. Rise-fall time, duration, repetition rate, and SLs were the same as those which characterized the stimuli employed to identify the SRMs in Part 1. Each listener received 120 trials-20 from each loudspeaker presented in an irregular order but never more than twice in succession.

\section{Results}

Table 3 details the localization performances of R.B. and A.E. Here, the apparent locations of the stimuli are plotted against their actual locations. As anticipated, R.B. performed significantly better than chance (56 correct responses in 120 presentations; $\mathrm{p}<.005$, one-tailed distribution); A.E. did not (p > .05, one-tailed distribution).

Table 3

Frequency Distribution of Apparent Locations Plotted Against Actual Locations of a 4.0-kHz-Wide Noise Band Centered at $7.5 \mathrm{kHz}$

\begin{tabular}{|c|c|c|c|c|c|c|c|c|c|}
\hline \multirow{3}{*}{$\begin{array}{c}\text { Actual } \\
\text { Loca- } \\
\text { tion }\end{array}$} & \multicolumn{9}{|c|}{ Apparent Location } \\
\hline & \multicolumn{6}{|c|}{ R.B. } & \multicolumn{3}{|c|}{ A.E. } \\
\hline & 345 & 330 & 315 & 300 & 285 & 270 & 300 & 285 & 270 \\
\hline 345 & 1 & 16 & 3 & & & & & 6 & 14 \\
\hline 330 & & 2 & 18 & & & & 4 & 7 & 9 \\
\hline 315 & & & 12 & 8 & & & 2 & 13 & 5 \\
\hline 300 & & & 14 & 6 & & & 2 & 11 & 7 \\
\hline 285 & & & & & 20 & & 1 & 8 & 11 \\
\hline 270 & & & & & 5 & 15 & & 3 & 17 \\
\hline
\end{tabular}

Note-R.B. and A.E. served as listeners.

\section{DISCUSSION}

The data of Part 1 firmly support the thesis that the apparent location of a narrow band of noise is influenced by its frequency composition, not its actual location. As the CF of the noise bursts was increased above $4.0 \mathrm{kHz}$, the apparent location of the sounds migrated toward the side of the functioning ear, that is, toward 270 deg azimuth. Our assumption that the spectral cues furnished primarily by the pinna are implicated in the apparent changes in the sounds' locations is strengthened by the results of Mehrgardt and Mellert (1977). They plotted transfer function from free-field to ear canal entrance. When the tonal frequency was increased from 4.0 to $9.0 \mathrm{kHz}$, the peak amplitude of the pinna response (near ear) moved from approximately 40 to $100 \mathrm{deg}$ from the midlinea physical event that parallels the SRM 1 exhibited by some of our listeners. In Part 2 of the present study, we searched for a direct relation between pinna amplification function and SRMs. By placing the microphone's diaphragm at the entrance of the ear canal, we reasoned that we were assessing the frequency response characteristics of the pinna. That no closer correspondence between the acoustic and behavioral measurements was obtained may be attributable to our restricted sample of the acoustic events. Sound pressure changes were recorded at only one small area at the ear canal entrance-roughly $1 \mathrm{~mm}$ square. Furthermore, considerable variability in location judgments was observed for most listeners, notwithstanding the fact that each test session consisted of a relatively large number of presentations. Hence, both the acoustical and behavioral data were contaminated to an unknown degree of experimental error. Yet in a fundamental way, our measurements of pinna amplification function are concordant with the overall localization judgments. As the apparent location of noise bursts moved toward the side loudspeakers ( 285 and $270 \mathrm{deg}$ ), so did the amplification of sounds from those loudspeakers increase relative to the amplification of sounds generated by the frontal loudspeakers ( 345 and $330 \mathrm{deg}$ ). The relative amplification, of course, was based on measurements at the ear canal entrance and not at the loudspeakers.

Part 3 represents an attempt to understand the basis for proficient monaural localization by testing listeners (A.E. and R.B.) whose SRMs and pinna amplification curves differed from one another in critical ways. The behavioral data collected on these two subjects strengthen the argument made by Butler and Flannery (1980) that accuracy on the task requires the spectral content of the stimulus to bridge adjacent SRMs. When the BW was fixed at $4.0 \mathrm{kHz}$ and centered at $7.5 \mathrm{kHz}$, this condition was met by R.B.'s constellation of location judgments, but not by A.E.'s 
(see Table 1). Concomitantly, sound pressure measurements on R.B. showed that the transition between his SRMs 1 and 2 was accompanied by an inversion in the pinna amplification function. In particular, the $1.0-\mathrm{kHz}$-wide noise band centered at $7.0 \mathrm{kHz}$, which appears to emanate from the side, independently of its actual location, was indeed amplified more when it was generated by the loudspeaker positioned at $270 \mathrm{deg}$ azimuth. The $1.0-\mathrm{kHz}$-wide noise band centered at $8.0 \mathrm{kHz}$, which appears to emanate from the frontal sector, independently of its actual location, was amplified more when it indeed was generated by loudspeakers located in the frontal sector (see Figure 3). Most likely, this phenomenon was responsible for R.B.'s localization performance when the noise bursts' BWs were only $2.0 \mathrm{kHz}(\mathrm{CF}$ was $7.5 \mathrm{kHz}$ ), for he was able to correctly locate the stimuli when their genesis was either from the front or from the side of the functioning ear (see Results, Part 1).

Our results suggest that only frequencies above the 6.0 - to $8.0-\mathrm{kHz}$ region are important for accurate monaural localization. Where to draw the lower frequency limit depends upon the specific configuration of a listener's pinna. We propose that possibility because (1) only for the noise bursts with higher CFs did the slope of the pinna amplification curve differ appreciably from zero, and (2) the spectral region critical for accurate localization is that which connects adjacent SRMs. These lower frequencies-4.0, 5.0 , and, for some listeners, $6.0 \mathrm{kHz}$-obviously do not.

Discarding the pinna amplification curves for noise bursts with low CFs, we propose the following scheme to account for the utilization of the spectral content of a stimulus for monaural localization: Given the pinna's structural configuration, a restricted frequency region within the spectrum is amplified relative to others. What part happens to be boosted most depends on the interaction between pinna configuration and the azimuth of the sound source. We propose that listeners are able to detect this boosted or dominant frequency region through its pitch-like quality. And, the apparent location of this complex sound is the spatial referent of its dominant frequency. Hence, under monaural listening conditions, perceiving the dominant frequency region of a complex stimulus by means of its pitch-like attribute is tantamount to locating it. The perception is immediate and without ratiocination.

In conclusion, we defined SRMs as a constellation of location judgments of narrow noise bands, influenced by the spectral content of the stimuli. We suggest that the pinna contributes to the clarity of the SRMs by differentially amplifying one spectral region over another, depending on the angle of the sound source vis-a-vis the interaural axis. Implicit in the discussion is the notion that SRMs are ultimately based on a neurological template, tonotopically organized, and upon which the monaural listener judges the direction of an impinging sound. Obviously, we have no data bearing directly on this issue, but should such a mechanism exist, the place principle of neurological organization, which is fundamental to visual and somatic space perception, could also account for much of the data on auditory space perception.

\section{REFERENCES}

Вцоom, P. J. Creating source elevation illusions by spectral manipulation. Journal of the Audio Engineering Society, 1977, 25, 560-565.

Butler, R. A. The influence of the external and middle ear on auditory discriminations. In W. D. Keidel \& W. D. Neff (Eds.), Handbook of sensory physiology (Vol. 5, Pt. 2): Auditory system. Berlin: Springer-Verlag, 1975.

Butler, R. A., \& Belendiuk, K. Spectral cues utilized in the localization of sound in the medial sagittal plane. Journal of the Acoustical Society of America, 1977, 61, 1264-1269.

Butler, R. A., \& Flannery, R. The spatial attributes of stimulus frequency and their role in monaural localization of sound in the horizontal plane. Perception \& Psychophysics, 1980, 28, 449-457.

Hebrank, J., \& Wright, D. Spectral cues used in the localization of sound sources in the median plane. Journal of the Acoustical Society of America, 1974, 56, 1829-1834.

Mehrgardt, S., \& Mellert, V. Transformation characteristics of the external human ear. Journal of the Acoustical Society of America, 1977, 61, 1567-1585.

(Received for publication September 11, 1980; revision accepted March 26, 1981.) 